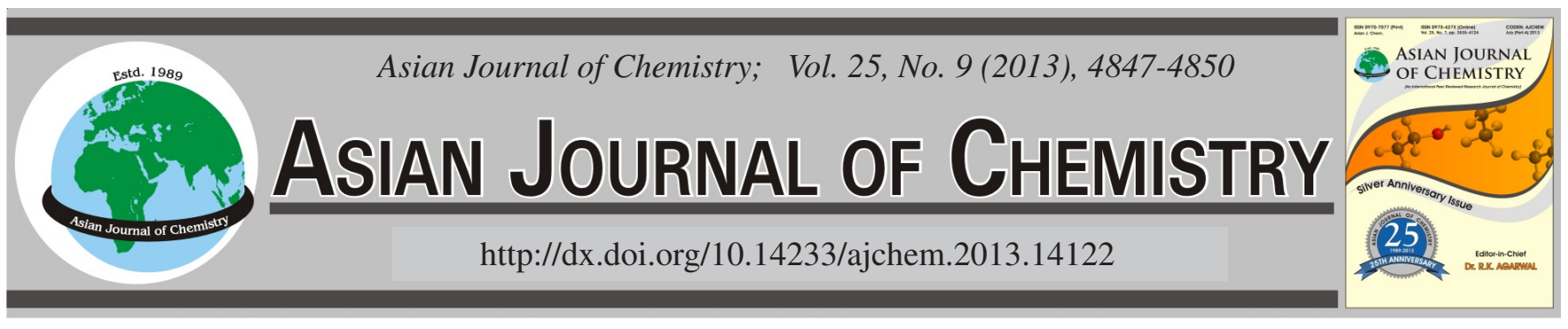

\title{
Synthesis of Superabsorbent Hydrogels Consisted of Pectin and Poly(methacrylonitrile) for Drug Delivery Systems
}

Mohammad Sadeghi", Anahita Gudarzi, Soudeh Safari, Hadis Shahsavari and Hossein Sadeghi

Department of Chemistry, Science Faculty, Arak Branch, Islamic Azad University, Arak, Iran

*Corresponding author: E-mail: m-sadeghi@iau-arak.ac.ir

\begin{abstract}
In the present paper, attention is paid to synthesize and evolution swelling behaviour of a superabsorbent hydrogel based on pectin and polyacrylonitrile. The physical mixture of pectin and polyacrylonitrile was hydrolyzed by $\mathrm{NaOH}$ solution to yield pectin-poly(sodium acrylate-co-acrylamide) superabsorbent hydrogel. the structure of the product was established using FTIR and SEM and TGA spectroscopies of initial substrates and superabsorbent hydrogel, respectively. The pectin-poly(sodium acrylate-co-acrylamide) hydrogel exhibited a pHresponsiveness character so that a swelling-deswelling pulsatile behaviour was recorded at $\mathrm{pHs} 1.6$ and 7.4.
\end{abstract}

Key Words: Pectin, Polyacrylonitrile, Superabsorbent, pH-responsiveness.

\section{INTRODUCTION}

Hydrogels, chemically or physically crosslinked threedimensional networks composed of hydrophilic polymers, can absorb and retain large amounts of aqueous fluids and the absorbed water is hardly removable even under some pressure. Due to excellent properties compared with traditional water absorbing materials, hydrogels have drawn much attention in a wide variety of fields such as drug delivery system, wound dressings, gel actuators, artificial organs, medical pharmaceuticals and contact lenses ${ }^{1-3} \cdot \mathrm{pH}$-sensitive hydrogels have attracted increasing attention due to their unique properties. Swelling of such hydrogels in the stomach is minimal and thus the drug release is also minimal. Due to increase in $\mathrm{pH}$, the swelling degree increases as the hydrogels pass down the intestinal tract. A variety of synthetic or natural polymers with acidic or basic pendant groups have been employed to fabricate $\mathrm{pH}$-sensitive hydrogels for getting the desired controlled release of drugs ${ }^{4}$. The use of natural polymers in the design of $\mathrm{pH}$ sensitive hydrogels has received much attention due to their excellent biocompatibility. The aim of this work was not only to characterize new type of superabsorbents, but also to evaluate the usefulness and feasibility of these polysaccharidebased superabsorbents for orally administered drug delivery system.

\section{EXPERIMENTAL}

Pectin (chemical grade, MW 50000) was purchased from Merck Chemical Co. (Germany). Polyacrylonitrile (PAN) was synthesized through a method mentioned in the literature ${ }^{5}$. All other chemicals were analytical grade and used without further purification duble distilled water was used for the hydrogel preparation and swelling measurements.

Hydrogel preparation: A facial one step preparative method was used for the synthesis of Pec-poly(sodium acrylateco-acrylamide) hydrogel, Pec-poly(NaAA-co-AAm), hydrogel. A general procedure for alkaline hydrolysis of Pec-PAN mixture was conducted as follows. Pectin $(0.50-1.33 \mathrm{~g})$ was added to a three-neck reactor equipped with a mechanical stirrer (Heidolph RZR 2021, three blade propeller type, 50-500 rpm), including $35 \mathrm{~mL}$ doubly distilled water. The reactor was immersed in a thermostated water bath. After complete dissolution of pectin to form a homogeneous solution, certain amount of sodium hydroxide $(0 / 25-2 / 5 \mathrm{~N})$ was added to the Pectin solution at desired temperature (alkalization temperature, $20-120^{\circ} \mathrm{C}$ ). The mixture was allowed to stir for certain times (alkalization times, 20-90 $\mathrm{min})$. The various amount of polyacrylonitrile (0.50$4.50 \mathrm{~g}$ ) was dispersed in the reaction mixture to saponify for certain times and temperatures (alkaline time and temperature). During the saponification $\mathrm{NH}_{3}$ gas was evolved and a colour change was observed from red to light yellow. This discoloration was an indication of the reaction completion. The pasty mixture was allowed to cool to room temperature and neutralized to $\mathrm{pH} 8$ by addition of $10 \mathrm{wt} \%$ aqueous acetic acid solution. Then the gelled product was scissored to small pieces and poured in ethanol $(200 \mathrm{~mL})$ to dewater for $5 \mathrm{~h}$. The hardened particles were filtered and dried in oven $\left(50^{\circ} \mathrm{C}, 10 \mathrm{~h}\right)$. After grinding, the powdered superabsorbent hydrogel was stored away from moisture, heat and light. 
Studies of the dynamic and equilibrium degree of swelling: The tea bag (i.e., a 100 mesh nylon screen) containing an accurately weighed powdered sample $(0.5 \pm 0.001 \mathrm{~g})$ with average particle sizes between 40-60 mesh (250-350 $\mu \mathrm{m})$ was immersed entirely in distilled water $(200 \mathrm{~mL})$ or desired salt solution $(100 \mathrm{~mL})$ and allowed to soak for $3 \mathrm{~h}$ at room temperature. The tea bag was hung up for $15 \mathrm{~min}$ in order to remove the excess fluid. The equilibrated swelling (ES) was measured twice using the following equation:

$$
E S(g / g)=\frac{\text { Weight of swollen gel }- \text { Weight of dried gel }}{\text { Weight of dried gel }}
$$

The accuracy of the measurements was $\pm 3 \%$.

pH-Sensitivity: pH-Sensitivity of the hydrogel was investigated in terms of swelling and deswelling of the final product at two basic ( $\mathrm{pH}$ 7.4) and acidic ( $\mathrm{pH}$ 1.6) solutions, respectively. Swelling capacity of the hydrogels at each $\mathrm{pH}$ was measured according to eqn. 1 at consecutive time intervals (0.5 h).

Instrumental analysis: Fourier transform infrared (FTIR) spectra of samples were taken in $\mathrm{KBr}$ pellets, using an $\mathrm{ABB}$ Bomem MB-100 FTIR spectrophotometer (Quebec, Canada), at room temperature. The surface morphology of the gel was examined using scanning electron microscopy (SEM). After Soxhlet extraction with methanol for $24 \mathrm{~h}$ and drying in an oven, superabsorbent powder was coated with a thin layer of gold and imaged in a SEM instrument (Leo, 1455 VP).

\section{RESULTS AND DISCUSSION}

At the first step of the formation of Pec-poly(MAN) hydrogel, hydroxyl groups of pectin substrate was converted to corresponding alkoxide ions using sodium hydroxide solution. Then, these macroalkoxides initiate crosslinking reaction between some adjacent polymethacrylonitrile pendant chains. This reaction leads to intermediate formation of naphthyridine cyclic structures (including imine, $-\mathrm{C}=\mathrm{N}-$, conjugated bonds) with deep red colour. The intermediate was then hydrolyzed using residual sodium hydroxide aqueous solution to produce hydrophilic carboxamide and carboxylate groups with a resulting colour change from red to light yellow. This sharp colour change was used as an indication to halt the alkaline treatment. However, incompletely hydrolyzed structures may also give rise to a few crosslinking points result in a loosely crosslinked network. It has been reported, in the case of H-SPAN, a maximum conversion of $70 \%$ of nitrile to carboxyl groups and the remaining $30 \%$ are amide groups ${ }^{6}$. In fact, details of the chemical processes and mechanism involved in H-SPAN synthesis are not yet well understood. For instance, the incomplete hydrolysis is interpreted as being related to steric and polar factors ${ }^{7}$. Oh et al. ${ }^{8}$ suggested that condensation might also occur between carboxyl and amide groups to form imide structures. Therefore, in the case of our hydrogel, Pecpoly(NaAA-co-AAm), we realized that precise control of the ratio is practically impossible.

Infrared spectroscopy was carried out to confirm the chemical structure of the hydrogel. Fig. 1 shows the FTIR spectra of Pec-PMAN physical mixture and the resulted hydrogel, Pec-poly(NaAA-co-AAm). The band observed at
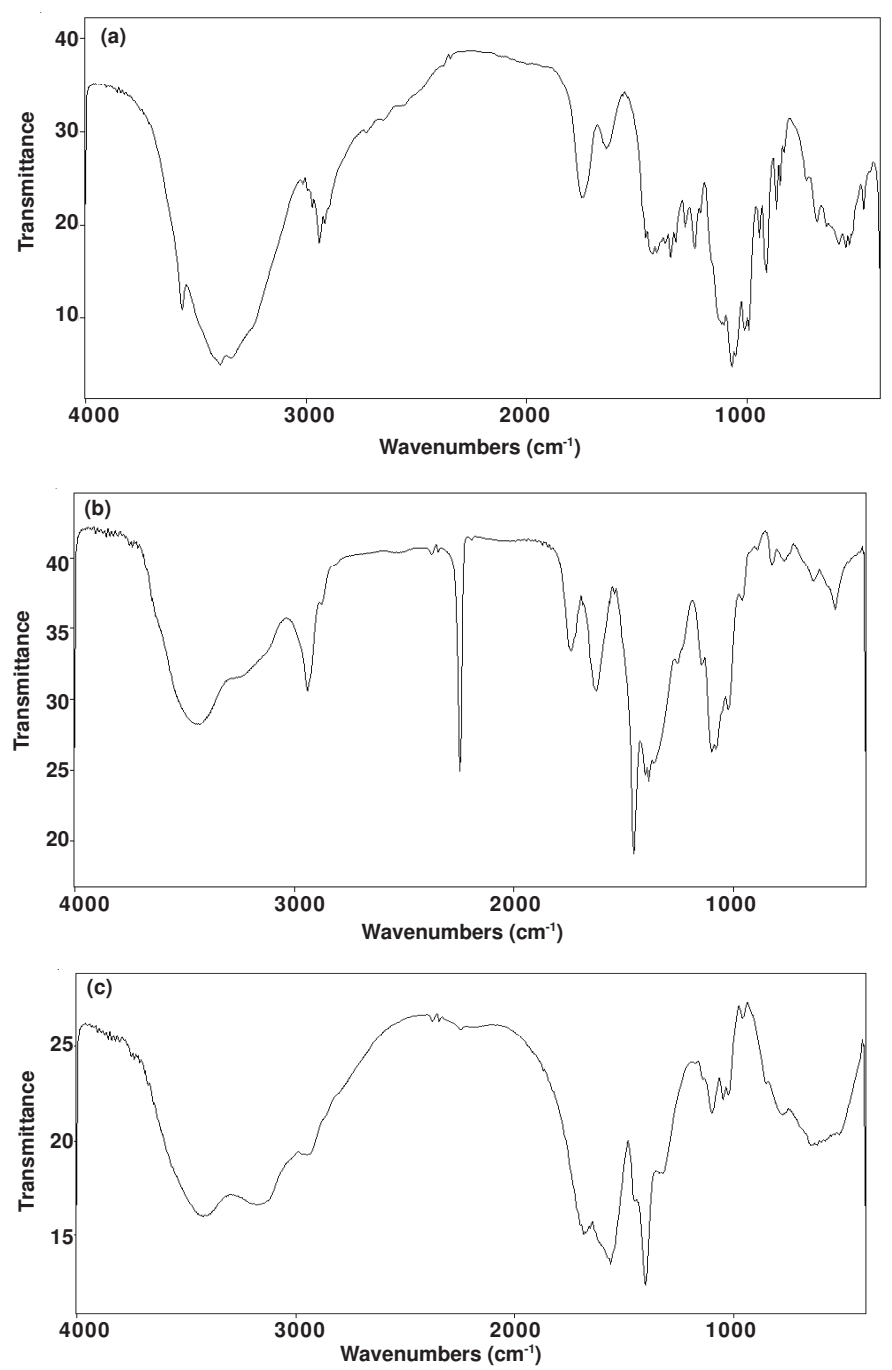

Fig. 1. FTIR spectra of pectin (a), pectin-g-PMAN (b) and H-pectin-gPAAm-co-PAcA hydrogel (c)

$2242 \mathrm{~cm}^{-1}$ can be attributed to stretching of -CN group of polymethacrylonitrile (Fig. 1b). The hydrogel comprise an pectin backbone with side chains that carry carboxamide and carboxylate functional groups that are evidenced by three new peaks at 1407, 1556 and $1675 \mathrm{~cm}^{-1}$ (Fig. 1c). These peaks attributed to $\mathrm{C}=\mathrm{O}$ stretching in carboxamide functional groups and symmetric and asymmetric stretching modes of carboxylate groups, respectively ${ }^{9}$. The stretching band of -NH overlapped with the $-\mathrm{OH}$ stretching band of the Pectin portion of the copolymer. As shown in Fig. 1c, after alkaline hydrolysis, most of the nitrile groups are converted to carboxamide and carboxylate groups.

To obtain an additional evidence of in situ crosslinking during alkaline hydrolysis, a similar reaction was conducted in absence of the polysaccharide. Since the resulted product became soluble, the crosslinks really formed between the alkoxide ions of pectin and the nitrile groups of PMAN. This fact practically proves that the starch hydroxyls are involved in the crosslinking.

Scanning electron microscopy: One of the most important properties that must be considered is hydrogel microstructure morphologies. The surface morphology of the samples was investigated by scanning electron microscopy. Fig. 2 shows 
an SEM micrograph of the polymeric hydrogels obtained from the fracture surface. The hydrogel has a porous structure. It is supposed that these pores are the regions of water permeation and interaction sites of external stimuli with the hydrophilic groups of the graft copolymers.
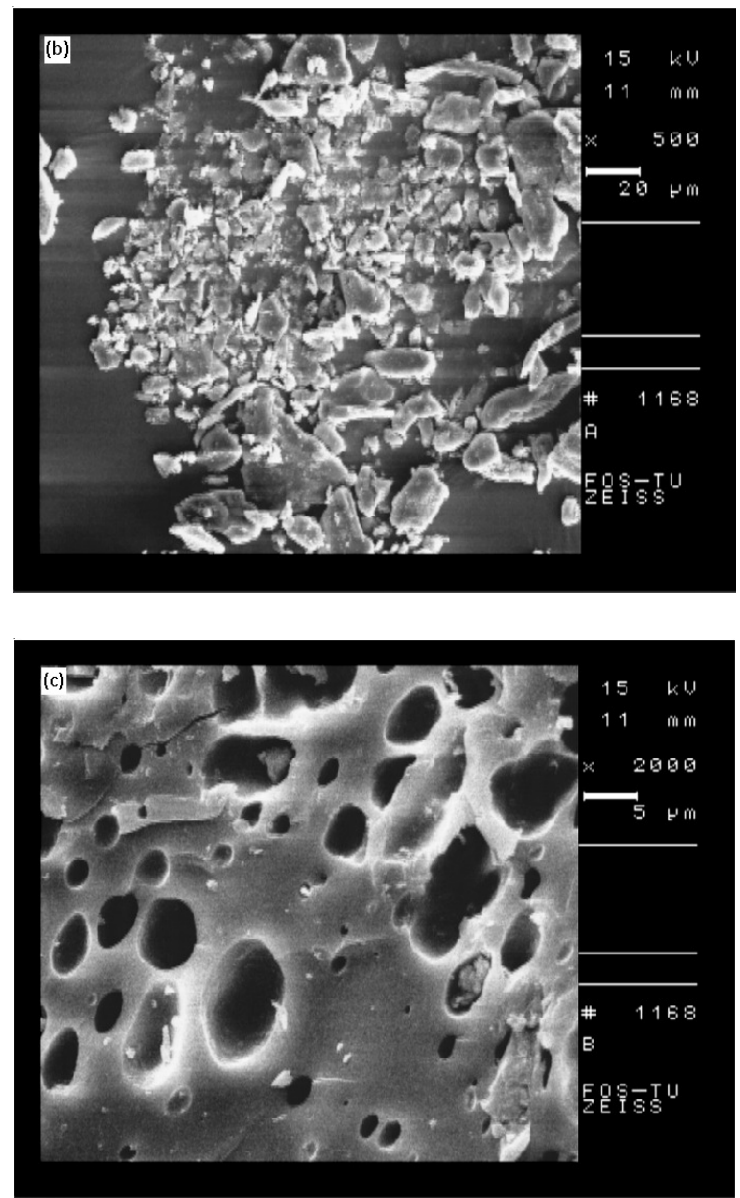

Fig. 2. SEM photograph of the pectin(a) and pectin-g-PMAN copolymer (b) H-pectin-g-PAAm-co-PAcA hydrogel (c)

Thermal analysis: Thermogravimetric analysis (TGA) was employed to thermally characterize the composite hydrogel in comparison with the raw pectin (Fig. 3). The thermal stability of the grafted pectin is improved as is obvious from the TGA curve. TGA of pectin (Fig. 3a) shows a weight loss in two distinct stages. The first stage ranges between 15 and $145^{\circ} \mathrm{C}$ and shows ca. $19 \%$ loss in weight. This may correspond to the loss of adsorbed and bound water. No such inflexion was observed in the TGA curve of $\mathrm{H}$ - pectin-g-PAAm-co-PAcA hydrogel (Fig. 3c). This indicated that the grafted copolymers were resistant to moisture absorption. The second stage of weight loss starts at $241{ }^{\circ} \mathrm{C}$ and continues up to $310{ }^{\circ} \mathrm{C}$ during which there was $57 \%$ weight loss due to the degradation of pectin. In general, degradation of native pectin is faster than that of grafted pectin. About $63 \%$ weight loss takes place in the temperature range of $240-385^{\circ} \mathrm{C}$ for pectin. In the $\mathrm{H}$ pectin-g-PAAm-co-PAcA hydrogel, a residual weight of $79 \%$ was observed at $325^{\circ} \mathrm{C}$. The appearance of these stages indicates the structure of pectin backbones has been changed, which might be due to the grafting of poly(AAm-co-AcA) chains. In general, the copolymer had lower weight loss than

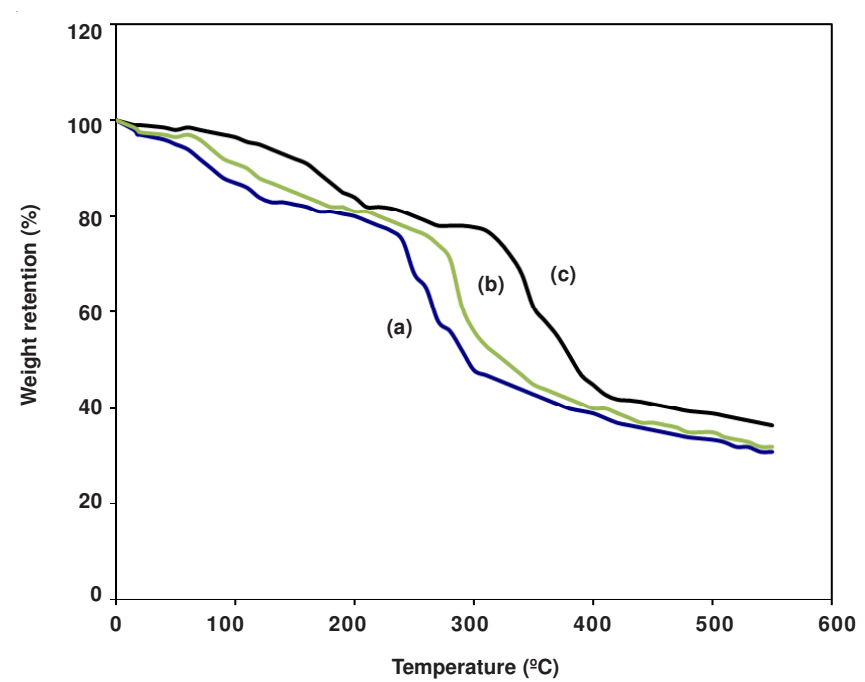

Fig. 3. TGA of pectin (a), pectin-g-PMAN (b) and H-pectin-g-PAAm-coPAcA hydrogel (c)

pectin. This means that the grafting of pectin increases the thermal stability of pectin in some extent.

Effect of pH on the swelling capacity: A pH-dependent swelling behaviour was observed at $25^{\circ} \mathrm{C}$ with changes in $\mathrm{pH}$ (Fig. 4). The equilibrium swelling (ultimate absorbency) of the superabsorbent composite was studied at various $\mathrm{pHs}$ ranged from 1-13. For preparing the $\mathrm{pH}$ media in these experiments, the stock $\mathrm{NaOH}(\mathrm{pH} 13)$ and $\mathrm{HCl}(\mathrm{pH} 1)$ solutions were diluted with distilled water to reach desired basic and acidic pHs, respectively.

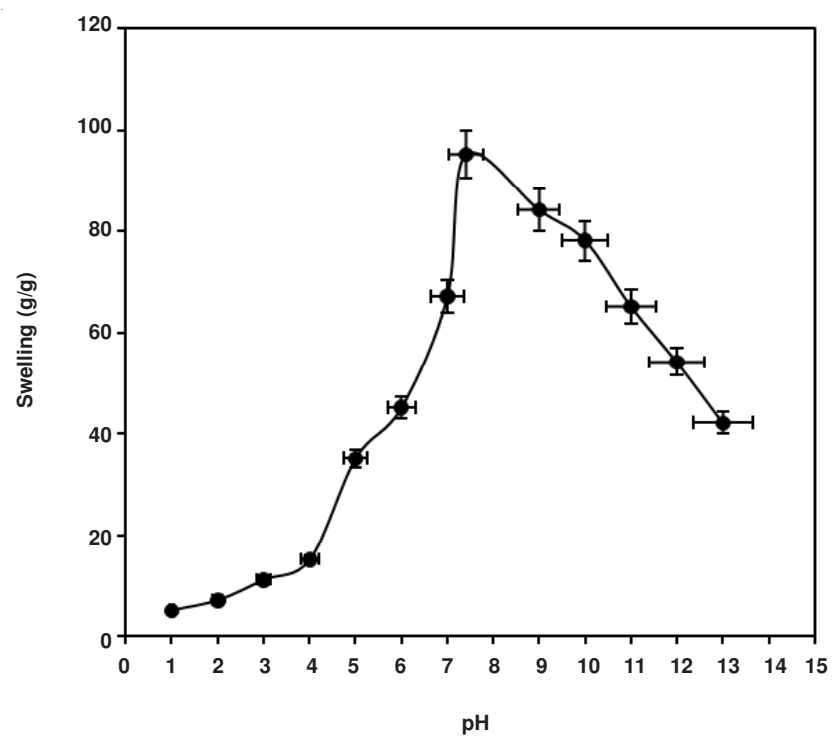

Fig. 4. pH-dependent swelling of the superabsorbent hydrogel

The swelling of the hydrogel composite is increased with increasing of the $\mathrm{pH}$ values from 1 to 7.4 , while it is decreased at $\mathrm{pHs}$ ranged of 7.4-12. The $\mathrm{pK}_{\mathrm{a}}$ of the carboxylic acid groups on backbone of polymer is $c a$. 4.5-5.5. When $\mathrm{pH}$ is less than $\mathrm{pK}_{\mathrm{a}}$, the $\mathrm{H}^{+}$ion strength will be high, which will effectively suppress the ionization of carboxylic acid groups. So, at acidic $\mathrm{pHs}$, the gel is neutral and flexibility of the polymeric chain is rather low. Carboxylic acid groups within the polymeric network is ionized to the $\mathrm{COO}^{-}$form and attract cations into 
the gel region to replace $\mathrm{H}^{+}$ions, since the $\mathrm{pH}$ of the environmental solution rises above its $\mathrm{pK}_{\mathrm{a}}$ value. This effectively increases the concentration of the free ions inside the composite. Therefore, because of the ionic swelling pressure increase, the composite tends to expand and thereby maximizes the repulsion between the ionized polycarboxylate groups.

The maximum water absorbency of the hydrogel was achieved at $\mathrm{pH}$ 7.4. At this point, all the $-\mathrm{COOH}$ groups are converted to the $-\mathrm{COO}^{-}$. Increasing of the anion density in the hydrogel, results in high swelling capacity. Beyond $\mathrm{pH} 7.4$, the swelling capacity is decreased. This can be attributed to the increase of the ionic strength and shielding effect (screening effect $)^{10}$. At high $\mathrm{pH}$, the $\mathrm{Na}^{+}$cations from $\mathrm{NaOH}$, shield the $-\mathrm{COO}^{-}$groups and prevent the perfect anion-anion repulsion.

pH-Responsiveness behaviour of the hydrogel: Since the hydrogels show different swelling behaviours at various $\mathrm{pHs}$, we investigated their $\mathrm{pH}$-reversibility in the solutions buffered at pHs 1.6 and 7.4 (Fig. 5). The figure shows a stepwise reproducible swelling change of the hydrogel at $25^{\circ} \mathrm{C}$ with alternating $\mathrm{pH}$ between 1.6 and 7.4. At $\mathrm{pH} 7.4$, the hydrogel swells up to $95 \mathrm{~g} / \mathrm{g}$ due to anion-anion repulsive electrostatic forces, while, at $\mathrm{pH} 1.6$, it shrinks within a few minutes due to protonation of carboxylate groups. This sharp swelling-deswelling behaviour of the hydrogels makes them suitable candidates for controlled drug delivery systems ${ }^{11,12}$.
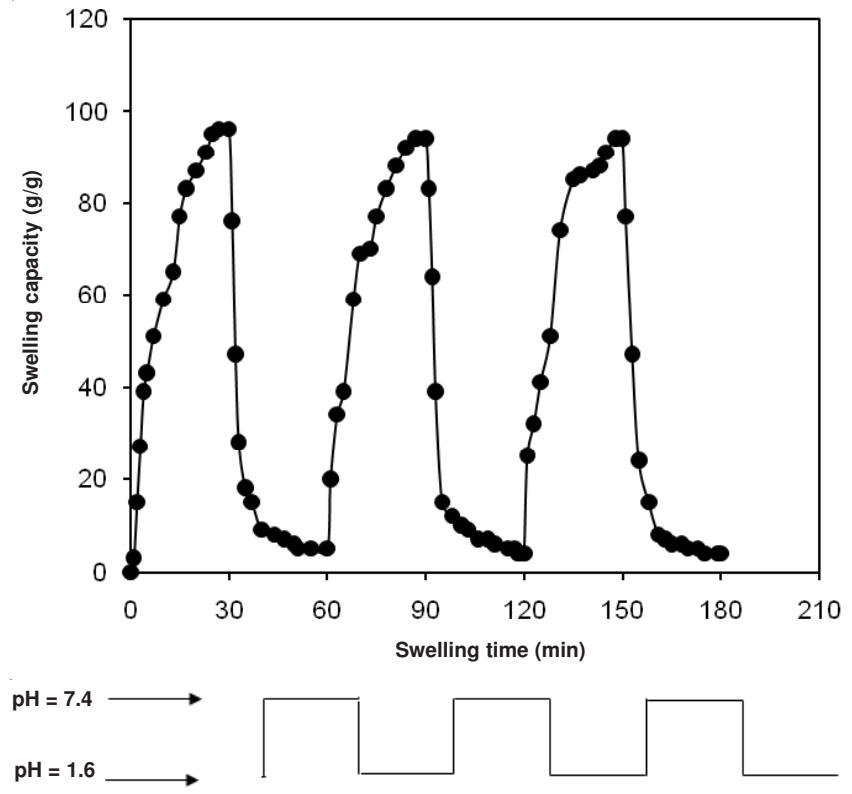

Fig. 5. On-off switching behaviour as reversible pulsatile swelling (pH 7.4) and deswelling ( $\mathrm{pH}$ 1.6) of the hydrogel. The time interval between the $\mathrm{pH}$ changes was $0.5 \mathrm{~h}$

\section{Conclusion}

The superabsorbent hydrogel, Pec-poly(MAN), was synthesized through alkaline hydrolysis of Pectin-PMAN physical mixture. The reaction of pectin alkoxide anions with nitrile groups of polymethacrylonitrile, forms crosslinking points and results in a three-dimensional network. Because a polymerization reaction is not involved, so there is no need to initiator, toxic and/or expensive monomer and crosslinker. Therefore, problems such as polymerization control, conversion loss and residual monomer are eliminated. Indeed, since no toxics material is used for the synthesis, this practical approach may be preferred to as a relatively "green process". The superabsorbent hydrogels exhibited high sensitivity to $\mathrm{pH}$, so that, several swelling changes of the hydrogel were observed in $\mathrm{pH}$ variations of a wide range (1-13). Ionic repulsion between charged groups incorporated in the gel matrix by an external $\mathrm{pH}$ modulation could be assumed as the main driving force responsible for such abrupt swelling changes. Furthermore, the reversible swelling-deswelling behaviour in solutions with acidic and basic $\mathrm{pH}$ makes the hydrogels a suitable candidate for controlled drug delivery systems.

\section{REFERENCES}

1. F.L. Buchholz and A.T. Graham, In: Modern Superabsorbent Polymer Technology. Wiley, New York (1997).

2. H. Cheng, J.L. Zhu, Y.X. Sun, S.X. Cheng, X.Z. Zhang and R.X. Zhuo, Bioconjug. Chem., 19, 1368 (2008).

3. L.Y. Chu, J.W. Kim, R.K. Shah and D.A. Weitz, Adv. Funct. Mater., 17, 3499 (2007).

4. L.Y. Chu, T. Yamaguchi and S.A. Nakao, Adv. Mater., 14, 386 (2002).

5. V. Crescenzi, L. Cornelio, C. Di Meo, S. Nardecchia and R. Lamanna, Biomacromolecules, 8, 1844 (2007).

6. H. Kranz and R. Bodmeier, Eur. J. Pharm. Sci., 34, 164 (2008).

7. I.C. Kwon, Y.H. Bae and S.W. Bae, Nature, 354, 291 (1991).

8. J.K. Oh, R. Drumright, D.J. Siegwart and K. Matyjaszewski, Prog. Polym. Sci., 33, 448 (2008).

9. J. Siepmann and N.A. Peppas, Adv. Drug Deliv. Rev., 48, 139 (2001).

10. K.S. Soppimath, T.M. Aminabhavi, A.M. Dave, S.G. Kumbar and W.E. Rudzinski, Drug Dev. Ind. Pharm., 28, 957 (2002).

11. M. Sadeghi and M. Yarahmadi, Orient. J. Chem., 27, 417 (2011).

12. M. Sadeghi and M. Yarahmadi, J. Chem., 27, 453 (2011). 